\title{
D-brane Gases and Stabilization of Extra Dimensions in Dilaton Gravity
}

\author{
Savas Arapoglu* \\ Department of Physics, Bog̃aziçi University, \\ Bebek, 34342, Istanbul, Turkey \\ Ali Kaya ${ }^{\dagger}$ \\ Feza Gürsey Institute, \\ Çengelköy, 81220, Istanbul, Turkey
}

(Dated: November 7, 2018)

\begin{abstract}
We consider a toy cosmological model with a gas of wrapped $D p$-branes in 10-dimensional dilaton gravity compactified on a $p$-dimensional Ricci flat internal manifold. A consistent generalization of the low energy effective field equations in the presence of a conserved brane source coupled to dilaton is obtained. It is then shown that the compact dimensions are dynamically stabilized in string frame as a result of a balance between negative winding and positive momentum pressures. Curiously, when $p=6$, i.e. when the observed space is three dimensional, the dilaton becomes a constant and stabilization in Einstein frame is also realized.
\end{abstract}

One of the main problems of string cosmology is to determine why the extra compact dimensions evolve differently from the observed three dimensions and remained comparatively very small. In [1], an intuitive mechanism was proposed to accommodate this difference where strings winding extra dimensions fall out of thermal equilibrium and stop the cosmological expansion. In [2-5], the arguments of [1] are quantified by demonstrating stabilization in Einstein and dilaton gravities using the energy momentum tensor for string winding and momentum modes on the torus (see also [6-21] for recent work on brane gas cosmology).

As pointed out in [6], strings may not be able to stabilize extra dimensions when topology is different than a torus. Considering, for example, a spherical internal space, there exists no stable winding strings in the spectrum. One would then wonder if the higher dimensional branes can play a role in these compactifications. In [6], it is shown that a gas of $p$-branes wrapping over a $p$ dimensional compact, Ricci flat, internal manifold can stabilize the volume modulus. As for strings, it turns out that there is a balance between the winding and the vibrational momentum modes. The results of $[6]$ are obtained in Einstein gravity and the purpose of this letter is to generalize the framework to dilaton gravity. Here, it is natural to consider a toy model with a gas of winding $D$ branes. Since dilaton couples to $D$-brane world-volume, it should be activated in this scenario.

Our first aim is to generalize the equations of motion in the presence of a conserved source coupled to dilaton. In string frame, the low energy effective action (we set $\left.H_{\mu \nu \rho}=0\right)$ is given by

$$
S=\int d^{10} x \sqrt{-g} e^{-2 \phi}\left[R+4(\nabla \phi)^{2}+e^{a \phi} \mathcal{L}_{m}\right]
$$

where we add a term to include the effects of matter governed by the Lagrangian $\mathcal{L}_{m}$. From this action, the field equations can be found as

$$
\begin{aligned}
R_{\mu \nu} & +2 \nabla_{\mu} \nabla_{\nu} \phi \\
& -\frac{1}{2}\left[R+4 \nabla^{2} \phi-4(\nabla \phi)^{2}\right] g_{\mu \nu}=e^{a \phi} T_{\mu \nu}, \\
R & +4 \nabla^{2} \phi-4(\nabla \phi)^{2}=F
\end{aligned}
$$

where $T_{\mu \nu}$ is the matter energy momentum tensor

$$
T_{\mu \nu}=-\frac{1}{\sqrt{-g}} \frac{\partial}{\partial g^{\mu \nu}}\left(\sqrt{-g} \mathcal{L}_{m}\right)
$$

and the extra contribution $F$ in (3) arises from the coupling of dilaton to $\mathcal{L}_{m}$.

The conservation formula $\nabla_{\mu} T^{\mu \nu}=0$ plays the role of matter field equations. To determine the unknown function $F$ we note the well known fact that (3) can be viewed as a consequence of (2) and the contracted Bianchi identity. Taking the divergence of (2) with $\nabla^{\mu}$, one finds:

$$
\left(\nabla_{\nu} \phi\right) F=(a-2) e^{a \phi} T_{\nu \lambda} \nabla^{\lambda} \phi .
$$

Although in general $F$ cannot be uniquely fixed by this constraint, in a cosmological context one can assume that

$$
\phi=\phi(t), \quad g_{t i}=0, \quad T_{t i}=0,
$$

where $t$ is the time coordinate and $i$ denotes a spatial direction. Under these conditions, (5) gives

$$
F=(a-2) e^{a \phi} T_{t t} g^{t t}=-(a-2) e^{a \phi} \rho .
$$

It is easy to see that (7) corresponds to the choice

$$
\mathcal{L}_{m}=-\rho,
$$

which is precisely the Lagrangian for hydrodynamical matter (see e.g. section 10.2 of [22]).

Let us take a $(1+m+p)$-dimensional space-time with the metric

$$
d s^{2}=-d t^{2}+d x^{i} d x^{i}+R^{2} d \Sigma_{p}^{2},
$$


and consider a $p$-brane wrapping over $\Sigma_{p}$. Here $i, j=$ $1, . ., m,(a, b=1, . ., p), \Sigma_{p}$ is a $p$-dimensional compact manifold and $R$ is a constant scale factor. The brane dynamics is governed by the usual action

$$
S_{p}=-T_{p} \int d^{p+1} \sigma \sqrt{-\gamma},
$$

where $T_{p}$ is the tension, $\gamma$ is the induced metric and the local brane coordinates are identified as $\sigma=\left(t, \Sigma_{p}\right)$. It is easy to see that the wrapped brane offers a stable classical configuration which solves the embedding equations derived from (10). Although it is not known how to quantize (10) exactly, for our purposes it is enough to employ an approximate scheme and simply consider small fluctuations around the classical background. It is clear that in this theory there are both winding and vibrational momentum modes in the spectrum. The energy of a winding mode is given by

$$
E=\left(n T_{p} \Omega_{p}\right) R^{p},
$$

where $n$ is the winding number and $\Omega_{p}$ is the volume of $\Sigma_{p}$. The spectrum of small vibrations can be found by expanding the action (10) to the second order in transverse fluctuation fields which yields free modes propagating in $\Sigma_{p}$. The energy spectrum is given by [6]

$$
E=\frac{\lambda_{k}}{R}
$$

where $-\lambda_{k}^{2}$ are the discrete eigenvalues of the Laplacian on $\Sigma_{p}$. For $D$-branes, there are additional $U(1)$ gauge fields living on the world-volume. At the linearized level, the transverse excitations obey free field equations and the energy spectrum is identical to (12).

The average pressure of a closed system can be found from the energy as a function of volume; $E(V)$. Recalling that in our case the volume modulus is fixed by $R^{p}$ one gets

$$
P=\left\{\begin{array}{cl}
-\left(n T_{p} \Omega_{p}\right) R^{p} & : \text { winding } \\
\lambda_{k} /(p R) & : \text { momentum }
\end{array}\right.
$$

Note that the winding and the momentum modes apply negative and positive pressures, respectively. For momentum modes, $1 / R$ dependence of the energy and the pressure can also be inferred from the uncertainty principle and the fact that the size of a compact direction is approximately equal to $R$.

For a non-interacting gas of such excitations, it is enough to sum over the modes with constant number densities to find the total energy and pressure. However, it is more natural to assume a state of thermal equilibrium $^{1}$ and consequently allow transitions between different energy levels. In that case, the mean number of a

\footnotetext{
${ }^{1}$ As discussed in [4], gravitons and photons offer an ideal can-
}

mode in the gas is proportional to the Boltzmann factor $e^{-\beta E}$. At very low temperatures the gas mainly consists of the lowest energy modes since the relative densities of other excitations are exponentially suppressed. In this case, one can use the expressions (11)-(13) with fixed quantum numbers (corresponding to lowest lying states) to obtain the average energy and pressure of the gas.

In a cosmological setting, one should replace (9) with

$$
d s^{2}=-d t^{2}+e^{2 B} d x^{i} d x^{i}+e^{2 C} d \Sigma_{p}^{2},
$$

where the metric functions $B$ and $C$ depend only on the proper time $t$. In this work we assume that $d \Sigma_{p}^{2}$ is Ricci flat. The scale factors for the observed and the internal spaces are defined by

$$
R_{o b}=e^{B}, \quad R_{\text {in }}=e^{C} .
$$

To find the energy momentum tensor from (11)-(13), the constant $R$ in these formulas should be replaced with the time dependent function $e^{C}$ and one should also divide by the total spatial volume $e^{m B+p C}$ to find the corresponding densities. This gives

$$
\begin{aligned}
T_{\hat{t} \hat{t}} & =T_{w} e^{-m B}+T_{m} e^{-m B-(p+1) C}, \\
T_{\hat{i} \hat{j}} & =0, \\
T_{\hat{a} \hat{b}} & =-T_{w} e^{-m B} \delta_{a b}+\frac{T_{m}}{p} e^{-m B-(p+1) C} \delta_{a b},
\end{aligned}
$$

where $T_{w}$ and $T_{m}$ are constants. It is easy to check that $T_{\mu \nu}$ is conserved; $\nabla_{\mu} T^{\mu \nu}=0$. As noted in [6], (16) implies an equation of state $p_{\hat{i}}=\omega_{i} \rho$, where for winding modes

$$
\omega_{i}=\left\{\begin{array}{cl}
-1: & \text { brane direction, } \\
0 & : \text { transverse direction, }
\end{array}\right.
$$

and for momentum modes

$$
\omega_{i}=\left\{\begin{array}{cl}
1 / p: & \text { brane direction, } \\
0: & \text { transverse direction, }
\end{array}\right.
$$

which is equivalent to radiation confined in the compact space.

Up to now our considerations are general and the formulas are valid for different brane types. To focus on $D$-branes we should fix the value of $a$. Since $D$-branes contribute first at disk order in string perturbation theory, their tension is inversely proportional to string coupling $g_{s}=e^{\phi}$. Therefore, one should set ${ }^{2}$

$$
a=1
$$

didate for a thermal bath which does not affect the dynamics of extra dimensions. In [6] we also observe that brane winding and momentum modes dominate the cosmology at late times in the presence of matter which has equation of state $p=\omega \rho$ with $\omega>0$. Therefore, the contribution of the thermal bath can be ignored in the following.

2 For fundamental strings the tension does not depend on $g_{s}$ and one should set $a=2$. In that case the field equations (2)-(3) agree with that of [2]. 
to have the correct $g_{s}$ dependence in (1). Recall that we have also

$$
m+p=9
$$

in string theory.

Using (16) in (2), (3) and (7) one can get the following equations (we take some linear combinations to simplify the expressions):

$$
\begin{aligned}
& \ddot{\phi}=-k \dot{\phi}+\frac{3-p}{2} F_{1}+2 F_{2}, \\
& \ddot{B}=-k \dot{B}+\frac{1}{2} F_{1}+\frac{1}{2} F_{2}, \\
& \ddot{C}=-k \dot{C}-\frac{1}{2} F_{1}+\frac{p+2}{2 p} F_{2}, \\
& k^{2}=m \dot{B}^{2}+p \dot{C}^{2}+2 F_{1}+2 F_{2},
\end{aligned}
$$

where

$$
\begin{aligned}
& k=m \dot{B}+p \dot{C}-2 \dot{\phi}, \\
& F_{1}=T_{w} e^{\phi-m B}, \\
& F_{2}=T_{m} e^{\phi-m B-(p+1) C},
\end{aligned}
$$

and dot denotes differentiation with respect to $t$. Eq. (22) can be viewed as a constraint on initial data and when it is obeyed at some $t_{0}$ it will be fulfilled during subsequent evolution.

We could not obtain the most general solution of these equations. Using, however, the following ansatz

$$
\begin{aligned}
& \phi=\phi_{1} \ln (t)+\phi_{0}, \\
& B=b_{1} \ln (t), \\
& C=C_{0},
\end{aligned}
$$

we find that (21) gives

$$
\begin{aligned}
& b_{1}=\frac{4}{m+3}, \quad \phi_{1}=\frac{2(m-3)}{m+3}, \\
& e^{\phi_{0}}=\frac{4(p+2) p}{T_{w}(p+1)(m+3)^{2}}, \\
& e^{(p+1) C_{0}}=\frac{(p+2) T_{m}}{p T_{w}},
\end{aligned}
$$

and (22) is identically satisfied.

In summary, we have the following power-law solution:

$$
\begin{aligned}
& d s^{2}=-d t^{2}+(t)^{8 /(m+3)} d x^{i} d x^{i}+e^{2 C_{0}} d \Sigma_{p}^{2}, \\
& e^{\phi}=e^{\phi_{0}}(t)^{2(m-3) /(m+3)} .
\end{aligned}
$$

It is obvious that in this background the internal dimensions are stabilized in string frame. Remarkably, when the observed space is three dimensional (i.e. when $m=3$ ) the dilaton does not depend on $t$ and the canonical Einstein frame becomes identical to string frame. Again for $m=3$, the expansion of the observed space is equivalent to the one for pressureless matter in standard cosmology. When $m>3$ the dilaton grows and at some point we enter the strong coupling regime in which the low energy field equations can no longer be trusted.

Apparently, (28) corresponds to special initial conditions and to be able to talk about dynamical stabilization we should consider more general initial data. To verify stabilization we did several numerical integrations with arbitrary initial conditions. Before presenting our findings let us discuss the following issue. The constraint (22) divides the solution space into two disjoint pieces according to the sign of $k$ defined in (23), since by (22) $k$ cannot vanish and thus its sign cannot alter in time. The sign is strongly correlated with the arrow of time in the problem. In all our numerical integrations we observe that when $k<0$ a singularity at finite proper time is encountered. These solutions can be thought to evolve backwards in time and hit the initial "big bang" singularity in finite duration. On the other hand all numerical integrations with $k>0$ yield smooth solutions and these can be thought to evolve forwards in time.

There is also a physically unexpected behavior for negative $k$. From the second equation in (21) we find that for $k<0$ and when initially $\dot{B}>0, \ddot{B}$ is always positive ${ }^{3}$ which implies an accelerated expansion for the observed space since the scale factor consequently obeys $\ddot{R}_{o b}>0$. This, however, contradicts with the anticipation that the winding and the momentum modes produce a deceleration by slowing down the expansion since from (17) and (18) they do not apply any pressure along the observed directions, i.e. they act like pressureless dust. This shows that (at least some) solutions with $k<0$ are not physically well-behaved.

Focusing, therefore, on initial data with $k>0$, we observe that in all numerical integrations the metric and the dilaton asymptotically approach (28). In figures 1, 2 and 3 we plot two illustrative examples for $m=3,2,4$, respectively. In these runs while $B$ eventually increases in time, $C$ performs damped oscillations around the constant value (27). For $m>3$ the dilaton grows, for $m<3$ it decreases and for $m=3$ it also oscillates around (26) which are damped in time. These results strongly indicate that (28) is a future asymptotic "fixed point" for (21) and (22).

To support these numerical findings analytically let us note that (21) can be viewed to describe a point particle in $(\phi, B, C)$ space. There are three forces acting on the particle: two of them are characterized by the functions $F_{1,2}$ and there is also a frictional force which is determined by the time dependent coefficient $k$. The fact that we restrict $k>0$ is crucial in identifying the frictional

\footnotetext{
${ }^{3}$ It is easy to see that the analogous argument for $k>0$ does not work.
} 

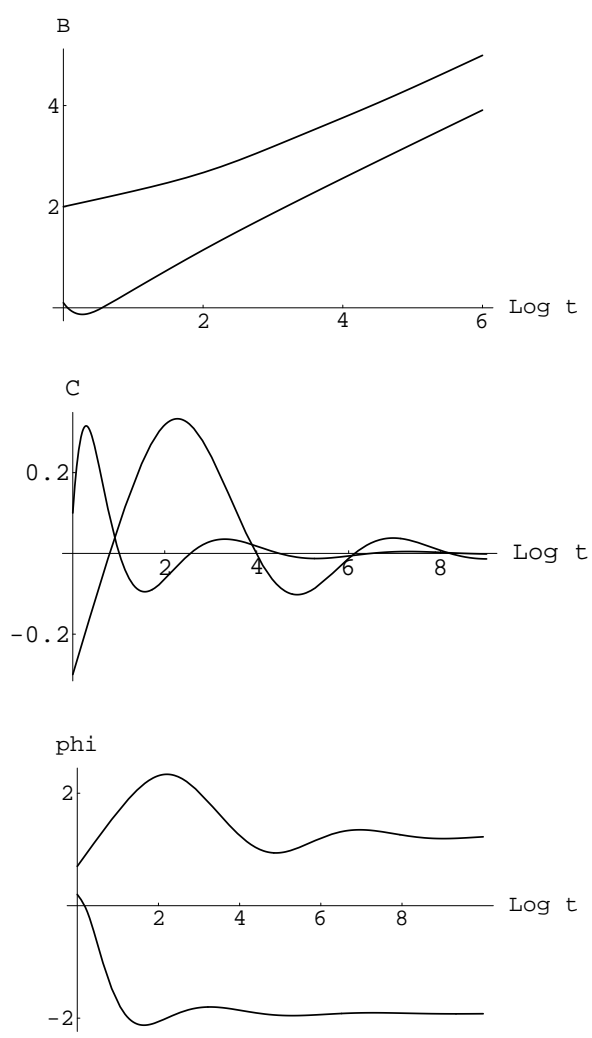

FIG. 1: $(m, p)=(3,6)$ : The graphs of $(B, C, \phi)$ for the following initial conditions $B(1)=(0.1,2.0), \dot{B}(1)=(-2.0,0.3)$, $C(1)=(0.1,-0.3), \dot{C}(1)=(1.7,0.4) \phi(1)=(0.2,0.7)$, $\dot{\phi}(1)=(-1.0,1.0)$, respectively. We choose $T_{w}=4$ and $T_{m}=3$ so that the internal dimensions are stabilized at $C=0$.

force $^{4}$. Ignoring friction for a moment, the third equation in (21) shows that the driving force along $C$ vanishes at $C=C_{0}$, where $C_{0}$ is given by (27). $C=C_{0}$ is a stable equilibrium point since when $C>C_{0}$ the net force is negative and when $C<C_{0}$ it is positive. For stabilization it is also crucial that the oscillations around $C_{0}$ are damped and this is exactly achieved by friction. Therefore, as $t \rightarrow \infty$ one expects $C \rightarrow C_{0}$ which proves stabilization.

On the other hand, using the asymptotic value $C_{0}$ in (21) one sees that the sum of the forces generated by $F_{1,2}$ in $\phi$ direction only vanishes for $m=3(p=6)$. In this case, due to the existence of friction the motion in $\phi$ should eventually stop and this explains the stabilization of dilaton when $m=3$. For $m \neq 3$, the net force never vanishes and thus $\phi$ will be time dependent. Similarly, the second equation in (21) shows that the net force along

\footnotetext{
${ }^{4}$ For $k<0$ there is an "anti-frictional" force. Not surprisingly this would destabilize the system and create a singularity. The existence of friction rather than anti-friction also signals that flow of time in the problem is correctly identified.
}
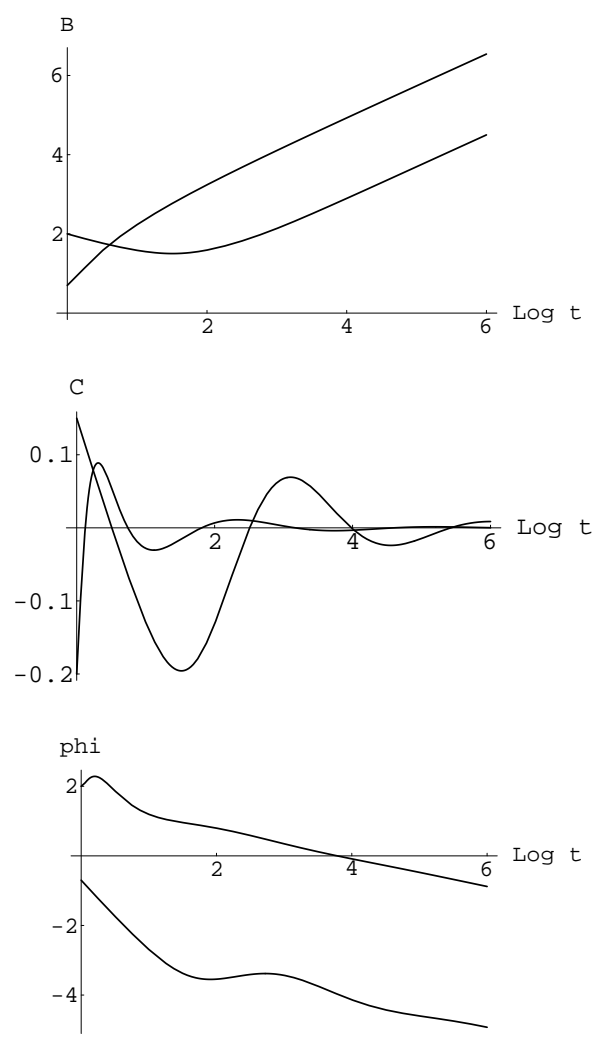

FIG. 2: $(m, p)=(2,7)$ : The evolution of $(B, C, \phi)$ for the initial data $B(1)=(0.7,2.0), \dot{B}(1)=(0.9,-0.5), C(1)=$ $(-0.2,0.2), \dot{C}(1)=(1.7,-0.3) \phi(1)=(2,-0.7), \dot{\phi}(1)=$ $(0.3,-2.1)$, respectively. We set $T_{w}=9, T_{m}=7$ and as a result $C_{0}=0$ from (27).

$B$ direction is never zero and thus $B$ cannot become a constant.

Technically, it is necessary for stabilization that $F_{1}$ and $F_{2}$ terms in the third equation in (21) have opposite signs. The value of $a$ is crucial for this condition to hold. In general the equation for $C$ takes the form

$$
\ddot{C}=-k \dot{C}-\frac{a}{2} F_{1}+\frac{2+(2-a) p}{2 p} F_{2} .
$$

For $a \leq 0$ both terms become positive and stabilization cannot be achieved. In the interval $0<a \leq 2$ the forces have opposite signs and for $a>(2 / p)+2$ the stabilization is again ruined. Remarkably for $D$-branes $a=1$, which is in the range that allows stabilization.

Concluding, in this paper we have presented a dynamical stabilization mechanism for the volume modulus in string theory based on the cosmological impact of a gas of $D$-branes. The model is obviously incomplete, however it reveals a generic dynamical behavior. It is not clear if this mechanism can be used in a realistic scenario to ensure moduli stabilization. One problem is that a straightforward generalization of Brandenberger and Vafa (BV) mechanism [7] implies all $p$-branes with 

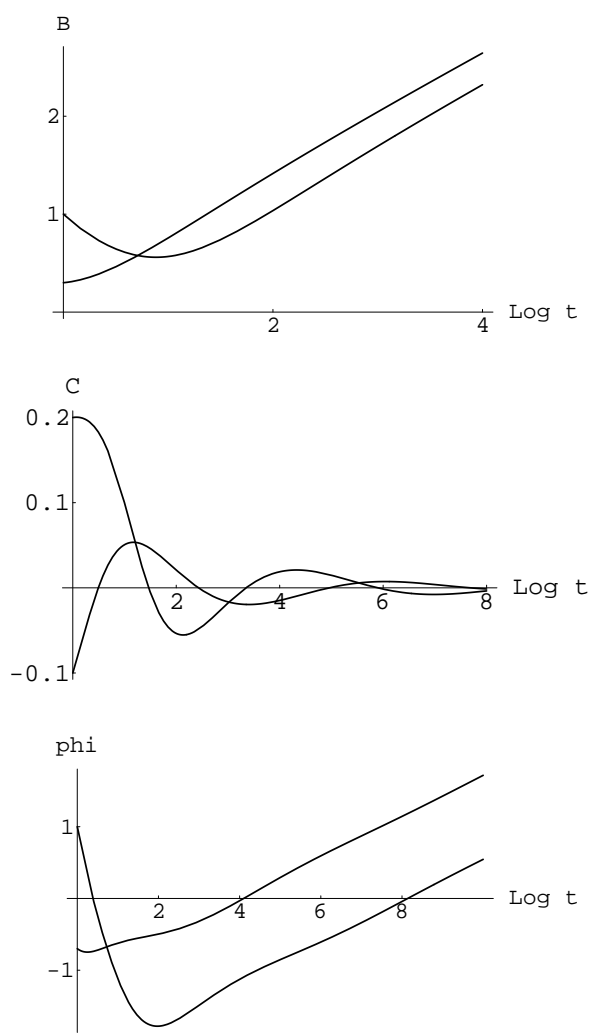

FIG. 3: $(m, p)=(4,5)$ : The results of numerical integrations with the initial conditions $B(1)=(1.0,0.3), \dot{B}(1)=$ $(-1.0,0.1), C(1)=(0.2,-0.1), \dot{C}(1)=(0.0,0.2), \phi(1)=$ $(1,-0.7), \dot{\phi}(1)=(-3.0,-0.4)$, respectively. We have also $T_{w}=7$ and $T_{m}=5$.

$p>2$ to annihilate in the early universe. Therefore, having a gas of higher dimensional branes at late times seems unlikely. On the other hand, as emphasized before, it seems that higher dimensional branes are needed for moduli stabilization in compactifications on topologically non-toroidal spaces like spheres or phenomenologically viable Calabi-Yau or G2 manifolds. Therefore, to have a realistic scenario involving brane gases it seems that we should either modify BV mechanism or improve the method based on winding strings to circumvent the topological restrictions.

* arapoglu@boun.edu.tr

† kaya@gursey.gov.tr

[1] R. Branderberger and C. Vafa, "Superstrings in the early universe", Nucl. Phys. B316, 391,(1989).

[2] S. Watson and R. Brandenberger, "Stabilization of Extra Dimensions at Tree Level", JCAP 0311 (2003) 008, hep- th/0307044

[3] S. Watson and R. Brandenberger, "Linear Perturbations in Brane Gas Cosmology", JHEP 0403 (2004) 045, hepth/0312097.

[4] S. P. Patil and R. Brandenberger, "Radion Stabilization by Stringy Effects in General Relativity and Dilaton Gravity", hep-th/0401037.

[5] T. Battefeld and S. Watson, "Effective Field Theory Approach to String Gas Cosmology", JCAP 0406 (2004) 001, hep-th/0403075.

[6] A. Kaya, "Volume Stabilization and Acceleration in Brane Gas Cosmology", JCAP 08 (2004) 004, hepth/0405099.

[7] S. Alexander , R.H. Brandenberger and D. Easson, "Brane gases in the early universe", Phys. Rev. D62, 103509, (2000), hep-th/0005212.

[8] D.A. Easson, "Brane Gases on K3 and Calabi-Yau Manifolds",Int. J. Mod. Phys. A18 (2003) 4295, hepth/0110225.

[9] R.H. Brandenberger, D.A. Easson and D. Kimberly, "Loitering Phase in Brane Gas Cosmology", Nucl. Phys. B623 (2002) 421, hep-th/0109165.

[10] R. Easther, B.R. Greene, and M.G. Jackson, "Cosmological String Gas on Orbifolds", Phys. Rev. D66 (2002) 023502, hep-th/0204099.

[11] S. Watson, R.H. Brandenberger, "Isotropization in brane gas cosmology", Phys. Rev. D67 (2003) 043510, hepth/0207168.

[12] T. Boehm and R.H. Branderberger, "On T-duality in brane gas cosmology", JCAP 0306 (2003) 008, hepth/0208188.

[13] R. Easther, B.R. Greene, M.G. Jackson and D. Kabat, "Brane gas cosmology in M-theory: late time behavior", Phys. Rev. D67 (2003) 123501, hep-th/0211124.

[14] S. H. S. Alexander, "Brane gas cosmology, M-theory and little string theory", JHEP 0310 (2003) 013, hepth/0212151.

[15] B.A. Bassett, M. Borunda, M. Serone and S. Tsujikawa, "Aspects of String-Gas Cosmology at Finite Temperature", Phys. Rev. D67 (2003) 123506, hep-th/0301180.

[16] A. Kaya and T. Rador, "Wrapped branes and compact extra dimensions in cosmology", Phys. Lett. B565 (2003) 19, hep-th/0301031.

[17] A. Kaya, "On Winding Branes and Cosmological Evolution of Extra Dimensions in String Theory", Class. Quant.Grav. 20 (2003) 4533, hep-th/0302118.

[18] T. Biswas, "Cosmology with branes wrapping curved internal manifolds", JHEP 0402 (2004) 039, hepth/0311076.

[19] A. Campos, "Late-time dynamics of brane gas cosmology", Phys. Rev. D68 (2003) 104017, hep-th/0304216.

[20] A. Campos, " Late cosmology of brane gases with a two-form field", Phys. Lett. B586 (2004) 133, hepth/0311144.

[21] J. Y. Kim, " Late time evolution of brane gas cosmology and compact internal dimensions", hep-th/0403096.

[22] V.F. Mukhanov, H. A. Feldman and R. H. Brandenberger, "Theory of Cosmological Perturbations", Phys. Rep. 215 (1992) 203. 